\title{
PRODUÇÃO DE CIANOBACTÉRIA EM REATOR ALTERNATIVO DE POLITEREFTALATO DE ETILENO (PET) EM QUATRO CORES DIFERENTES
}

\section{PRODUCTION OF CYANOBACTERIA IN ALTERNATIVE REACTOR POLYETHYLENE TEREPHTHALATE (PET) IN FOUR DIFFERENT COLORS}

\author{
Jéssica Mazetto de Araujoํ, Regina de Oliveira Moraes Arruda²
}

\begin{abstract}
RESUMO
Neste trabalho será realizada a produção da cianobactéria em reatores alternativos, tipo garrafa de Politereftalato de etileno (PET) em quatro cores diferentes, para verificar se há influência da cor no crescimento. $O$ experimento foi feito em duplicata para (oito garrafas com quatro cores diferentes de garrafa PET) no decorrer de 22 dias, teve influência igual da luz sobre todas as garrafas, agitação na mesma velocidade e mesmo aquecimento dentro da estufa, com mesma quantidade de meio de cultura e inóculo para todas as garrafas. A Arthrospyra (Spirulina) sp teve diferente nível de crescimento em cada cor diferente de garrafa de Politereftalato de etileno (PET). A coloração dos reatores afeta o crescimento e o desenvolvimento da Arthrospyra (Spirulina). Entre as quatro colorações de reatores estudadas a de cor vermelha é a mais eficiente na produção de Arthrospyra (Spirulina). A cor vermelha atingiu um pico maior de crescimento e seu tempo de vida se mostrou maior, portanto a cor vermelha mostrou exercer influência sobre o crescimento da Arthrospyra (Spirulina) sp, podendo assim vir a ser utilizada na produção em alta escala.
\end{abstract}

PALAVRAS-CHAVE: Arthrospyra (Spirulina) sp. Fermentação. Bioprocesso.

\begin{abstract}
In this study, we conducted the production of cyanobacteria in alternative reactors, type of polyethylene terephthalate bottle (PET) in four different colors, to check the influence of color on growth. The experiment was performed in duplicate (eight bottles with four different colors of PET bottles) during 22 days, all bottles had the same light influence, the same stirring speed and the same heat inside the greenhouse, with the same amount of culture medium and inoculum for all the bottles. The Arthrospyra (Spirulina) sp had a different level of growth in each different polyethylene terephthalate (PET) bottle color. The coloring of the reactors affects the growth and development of Arthrospyra (Spirulina). Among the four colorings reactors studied, the red color is the most efficient in the production of Arthrospyra (Spirulina). The color red has reached a higher growth peak and its lifetime was larger, so the red color exerted an influence on the growth of Arthrospyra (Spirulina) sp, may come to be used in large-scale production.
\end{abstract}

KEYWORDS: Arthrospyra (Spirulina) sp. Fermentation. Bioproces.

\footnotetext{
1 Graduada em Farmácia pela Universidade UNG.

${ }^{2}$ Docente do Mestrado em Análise Geoambiental. Universidade UNG.
} 


\section{INTRODUÇÃO}

A microalga Arthrospyra (Spirulina) era utilizada como produção de alimento pelos povos antigos que habitavam em margens de lagos da África e do México (VONSHAK, 1997)

Algas têm sido usadas como alimento humano, desde épocas imemoráveis, em várias partes do mundo: Índia, China, México, América do Norte, Japão, Peru e outras. Talvez as mais antigas e primitivas formas de vida na terra. Foram encontrados microrganismos fossilizados cerca de 3,5 bilhões de anos (SATO, 2009).

As microalgas pertencem a um grupo heterogêneo, são aquáticas e geralmente unicelulares apresentando a mesma composição química em toda a biomassa, sua característica tem a presença de pigmentos, as microalgas são compostas de espécies eucarióticas ou procarióticas, desde o período em que surgiram no planeta (RAVEN; EVERT; EICHHOM, 2001). A microalga cresce naturalmente (HENRIKSON, 1994), sua vantagem é á rapidez como elas se reproduzem (BOROWITZKA, 1995).

Arthrospyra (Spirulina) pertence à divisão Cyanophyta, algas verde-azuladas, também chamadas de cianobactérias. Pertence a classe das cianofíceas. Sua coloração verde escura deve-se aos pigmentos naturais: clorofila (verde), ficocianinas (azul), e carotenoides (laranja). É um alimento de fácil digestão, rapidamente absorvido e com a capacidade de proteger o nosso organismo. A Arthrospyra (Spirulina) se concentra B-caroteno cerca de 10 vezes mais que a cenoura, além de rica fonte de vitaminas do complexo B (SILVA; MURA; AYOUB, 2007).

Arthrospyra (Spirulina) pode ser produzida a partir de água do mar, de lagos salino ou de águas oriundas de sistemas de tratamento de efluentes. Como as cianobactérias são microrganismos fotossintetizantes, a influência da incidência de luz nos cultivos mostrou-se de extrema importância (HENRIKSON, 1994).

A Arthrospyra (Spirulina) tem grande interesse nutricional para a produção de biomassa, possuem um elevado teor protéico (60- 65\% para biomassa de $\mathrm{Ar}$ throspyra (Spirulina)) (SATO, 2009). Também estão presentes outros fito nutrientes, como ácido graxo essencial ácido y-linolênico [GLA], antioxidantes, sulfolípides, glicolípides e polissacarídeos (SILVA; MURA; AYOUB, 2007).

\section{OBJETIVOS}

Produção de cianobactéria (Arthrospyra (Spirulina) platensis) em reator alternativo, tipo garrafa PET, em quatro diferentes cores. - Produção de cianobactéria em reator alternativo usando o meio padrão Paoletti (1975).

Verificar a viabilidade de produção da cianobactéria em reator alternativo (garrafa pet.).

\section{REVISÃO DA LITERATURA}

A Arthrospyra (Spirulina) platensis é uma microalga filamentosa que habita em Vários lugares como "solo, areais, pântanos, lagos alcalinos, água salobras, marinhas doces". Através da fotossíntese, converte os nutrientes em matéria celular e também libera oxigênio (BERTOLIN et al., 2005).

Esta microalga pertence ao grupo Cyanobacterium, São organismos unicelulares fotoautótrofos (as células obtém energia da luz e do carbono e o carbono do $c 02$ do ar produzindo então, através da fotossíntese, suas biomoléculas) (ARRUDA et al., 2009; FRANCO et al., 2013).

Atualmente, utiliza-se Arthrospyra (Spirulina) platensis, como suplemento alimentar e também como um complemento nutricional (BRASIL, 1995).

A Arthrospyra (Spirulina) também tem suas vantagens no que se trata de propriedade de saúde. Características da Arthrospyra (Spirulina), "funcionais podem ser classificados em grupos tais como": alimentos sulficados e nitrogenados, pigmentos, próbioticos e prébioticos, vitaminas e fibras, proteínas, lipídios, acido graxos e compostos fenólicos. Essas características da Arthrospyra (Spirulina) têm aplicações clinicas podendo ter o seu efeito terapêutico em: Hiperlipidenia, Obesidade, Diabéticos Mellitus, Sistema Imunológico, Hipertensão Arterial, Toxicidade Renal, Radio protetor, Câncer, Antivirais e o principalmente em Desnutrição (AMBROSI et al., 2008). Além de, a Arthrospyra (Spirulina) platensis ser utilizada como suplemento alimentar pelos seres humanos ela também se mostrou eficaz em relação à dieta de tilápia (peixe).Uma microalga que tem uma enorme potencial para enriquecimento, em tilápia (peixe) e Camarão (crustáceo) os alimentos que contenha ração com Arthrospyra (Spirulina) platensis, "Foi observado um aumento na sua resistência" ou seja (atividade fagocitária) contra as bactérias Vibrio harveyi, Escheri- 
chia coli, Salmonella, Typhimurium, Bacillus subtilis esta resposta deu-se principalmente a presença de grupos lipopolissacaríedeos e peptideoglicanos que foi obtidos através da alimentação com a microalga Arthrospyra (Spirulina) platensis (MOREIRA et al., 2010).

"Durante o século XX" alguns pesquisadores e também produtores comerciais desenvolveram varias tecnologia de cultivo para poder produzir biomassa de microalgas, por exemplo, a Arthrospyra (Spirulina) platensis em tanques abertos e em fotobiorreatores fechados. O cultivo dessa microalga esta gradativamente em todo o mundo. A biomassa produzida é utilizada em diversas aplicações como, por exemplo, "produção de proteína unicelular, lipídeos, carotenóides, clorofila, enzimas, ésteres, antibióticos, hidrocarbonetos e vitaminas." Porém a principal aplicação da biotecnologia microalga é na produção de suplemento alimentar (BERTOLDI; SANT'ANNA; OLIVEIRA, 2008).

A cianobactéria do gênero Arthrospyra (Spirulina) platensis pode sofrer algumas, variações em seu crescimento isso é de acordo com o meio em que é cultivada, utilizando as condições do meio externo. Através da manipulação das condições de cultivo pode-se estimular a biossíntese de vários compostos, além de que pode também controlar estas variáveis, com o intuito de diminuir o custo do processo de produção (MEZZOMO et al., 2010).

As microalgas elas podem utilizar, como fonte de nitrogênio o nitrato. Essa biomassa que é produzida poderá ser usada como ração animal ou produção de energia e até mesmo fertilizantes, ou para poder produzir produtos de química fina (SASSANO et al., 2002).

A muitos fatores que pode influenciar o crescimento do gênero Arthrospyra (Spirulina), entre eles a fonte de nitrogênio. A falta de nitrogênio pode afetar o crescimento, sendo assim influencia a composição de lipídeos e "leva um decréscimo na produção de ficocianina e clorofila, " e consequente perda de suas características de cor verde azulado (SANTOS; MACEDO; ALEGRE, 2003).

O nitrogênio é um elemento muito importante para o metabolismo das microalgas como Arthorospira, que contribui muito para a formação de proteínas. Com a redução na quantidade de nitrogênio no meio de cultura pode possibilitar que os lipídeos e também os carboidratos seja sintetizados preferencialmente porem já com o aumento na quantidade ou na concentração de nitro- gênio no meio de cultura pode-se elevar o seu teor de clorofila na biomassa da microalga. Foi observado que com o conteúdo de clorofila na Arthrospyra (Spirulina) platensis (que em experimento cuja sua concentração de KNO3 variou de 0,003 a $0,1 \%$ no seu meio de cultura) atingiu o teor Maximo de $0,5 \%$ no cultivo com $0,1 \%$ de KNO3. No entanto em outro estudo, houve uma redução da taxa de fotossintética da microalga Chlorella sp. De acordo com a diminuição da fonte de nitrogênio do meio de cultura, a concentração de teor de nitrogênio também pode afetar outro composto da microalga. Quando se utiliza a fonte de nitrogênio que apresenta a forma de nitrato, a microalga vai realiza um gasto celular energético e para poder reduzir esse íon o nitrito através da enzima nitra-redutase. Que posteriormente, vai ocorrer outra redução pelo nitrito-redutase gerando a amônia que é uma forma de nitrogênio utilizado pela alga em seu metabolismo. (BERTOLDI; SANT'ANNA; OLIVEIRA, 2008; MULITERNO et al., 2005).

Depois do carbono o nitrogênio é um nutriente que contribui para a produção de biomassa. Uma resposta típica da limitação de nitrogênio é a sua descoloração das células, ou seja, (diminuição das clorofilas e um aumento de carotenóides), e de acúmulo de compostos de carbono inorgânico como, por exemplo, polissacarídeo, sua maior parte encontra-se na forma de nitrato NO-3 e muitas vezes na forma de amônia $\mathrm{NH}+4$ e também uréia. Há uma grande variedade de compostos orgânicos de $\mathrm{N}$ que são utilizados por algas, e que pode servir côo a única fonte de nitrogênio do $\mathrm{N}$-amônia é frequentemente a $\mathrm{N}$-fonte preferida para os micro-organismos e a através da assimilação do NO-3 ou do NH+4 é que esta relacionado ao $\mathrm{pH}$ dos meios de crescimento. Algumas cianobactérias e capas de utilizar o nitrogênio elementar pela redução do $\mathrm{N} 2$ a $\mathrm{NH}+4$, um processo catalisado pela nitrogênase da enzima. Embora a quantidade significativa pudesse ser fixa em uma base extensiva, tais como plantações de arroz ou em ecossistemas naturais as quantidades são demasiadas para o sistema de produção das algas, da taxa elevada onde $\mathrm{N}$ esta limitada e é altamente improvável e a $\mathrm{N}$-fixação que se aplicaria somente aos sistemas de produção extensivos. A consideração importante da biotecnologia das algas destina-se a obter altos rendimentos e assegurar a fonte adequada desse nutriente tão importante (RICHMOND, 2004). 


\section{MATERIAL E MÉTODOS}

Materiais

Reator: reator alternativo: garrafa $300 \mathrm{~mL}$ de Politereftalato de etileno (PET), 8 garrafas em 4 cores diferentes, 2 azul, 2 vermelhas, 2 transparentes e 2 verdes, a cor se deu por 8 garrafas em papel celofane de 4 cores diferentes.

Estufa: a agitação ocorreu dentro da estufa (onde tinha suporte para as garrafas), para manter as células em suspensão no meio líquido, prevenindo assim a sedimentação de nutrientes e fornecendo a célula igual distribuição da luz, garantindo assim a homogeneização da cultura. Neste experimento a agitação se deu em 120rpm.

Iluminação: Artificial (a pesquisa será desenvolvida dentro da estufa onde possui suporte para a lâmpada no canto esquerdo do alto da estufa). A disponibilidade das garrafas frente à intensidade da luz se deu em forma de rodízio, a cada medição ocorria à troca de lugares da garrafa dentro da estufa, assim todas as amostras tiveram a mesma influência da luz. A temperatura se deu em $30^{\circ} \mathrm{C}$ e no tempo de 22 dias.

Espectrofotômetro e Cubetas de $1 \mathrm{~cm}$ : verificação do crescimento da Arthrospyra (Spirulina). A faixa de crescimento será acompanhada através de biomassa produzida no decorrer dos dias e leituras periódicas em espectrofotômetro a $560 \mathrm{~nm}$. A cinética do crescimento será feita no período de 22 dias.

Foi utilizado, (Espectrofotômetro microprocessado Analyser), balança semi-analítica Gehaka BK 400 II, funil, Proveta, Pipeta graduada de $1 \mathrm{~mL}$ e $5 \mathrm{~mL}$, Espátula, Papel manteiga, Celofane, Papel filtro, Erlenmeyer, microrganismo Arthrospyra (Spirulina) platensis e meio de cultura.

\section{Método}

Inicialmente foi feito o meio de cultura, contendo: $\mathrm{NaHCO} 3$ (1,84g), NaCl $(3,76 \mathrm{~g}), \mathrm{Na} 2 \mathrm{SO} 4(1,0 \mathrm{~g}), \mathrm{K} 2 \mathrm{H}-$ PO4 (17,78g), Na2CO3 (30,30g), CaCl2.2H2O $(0,1)$, MgSO4 $(0,5)$. Acrescentar $2 \mathrm{~L}$ de água para cada meio e dissolver e após, colocar $2 \mathrm{~mL}$ de SOL-Fe.EDTA e SOL micronutrientes.

Também foi preparada a Solução do Fe-EDTA com: EDTA-Na 29,8g e FeSO4.7H2O 24,9 g; q.s.p. H2O para $1000 \mathrm{~mL}$, e a solução de microelementos com (mg/L): $\mathrm{SnCl} 2.2 \mathrm{H} 2 \mathrm{O}$ (304); $\mathrm{MnCl} 2.4 \mathrm{H} 2 \mathrm{O}$ (173); $\mathrm{CoCl} 2.6 \mathrm{H} 2 \mathrm{O}$ (194); $\mathrm{SeCl} 2.2 \mathrm{H} 2 \mathrm{O}$ (146); BaCl2.2H2O (2.486); LiCl (293); CuSO4.5H2O (186); NiSO4.6H2O (717); NaMoO4.2H2O (121), e H2O (q.s.p. 1000 mL).

Em cada garrafa foi colocado $240 \mathrm{~mL}$ de meio de cultura e $10 \mathrm{~mL}$ de inoculo Arthrospyra (Spirulina) platensis.

\section{MONTAGEM DO EXPERIMENTO}

O experimento foi montado no laboratório por duas vezes, dentro de uma estufa sob iluminação, agitação a $120 \mathrm{rpm}$ e temperatura adequadas de $30^{\circ} \mathrm{C}$, as garrafas foram utilizadas em duplicatas, embrulhadas em papel celofane de 4 cores distintas. As garrafas de Politereftalato de etileno (PET) com $240 \mathrm{~mL}$ de meio e $10 \mathrm{~mL}$ de inóculo, foram colocadas na estufa. O processo fermentativo foi de 22 dias. As amostras foram lidas em Espectro fotômetro no comprimento de onda de 560nm e acertado o zero com água.

\section{RESULTADOS}

Os resultados foram verificados através de leitura de absorbância no decorrer do experimento (22 dias) nos diferentes reatores alternativos de Politereftalato de etileno (PET). Foram estudadas quatro diferentes cores: transparente, azul, verde e vermelho. Nos quadros 1 e 2 apresentamos as leituras de absorbância.

Quadro 1. Média de Crescimento da Arthrospyra (Spirulina) sp (absorbância) no decorrer dos dias dos 22 dias de experimento em cada cor de reator

Table 1. Average Growth of Arthrospyra (Spirulina) $s p$ (absorbance) over the days of the 22 days of experiment in each color of reactor

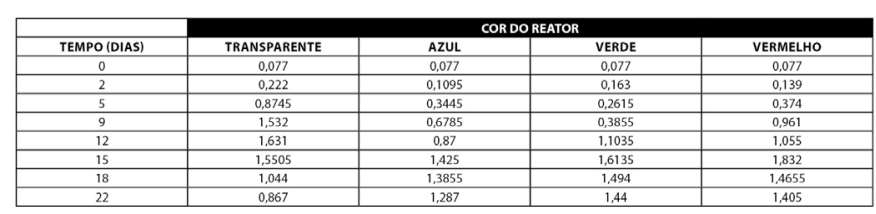


V.4, N.1, 2020

Quadro 2. Média de Crescimento da Arthrospyra (Spirulina) sp (absorbância) no decorrer dos dias do $2^{\circ}$ experimento em cada cor.

Table 2. Average Growth of Arthrospyra (Spirulina) $s p$ (absorbance) during the days of the 2nd experiment in each color.

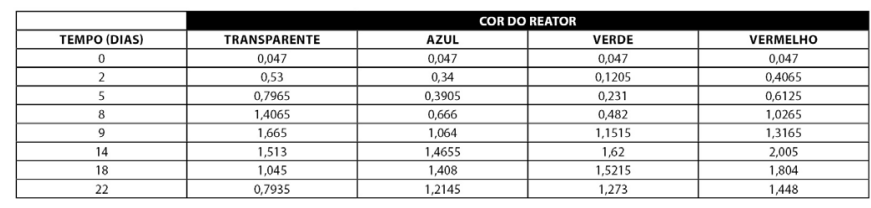

Nos gráficos 1 e 2 apresentamos a evolução do crescimento Arthrospyra (Spirulina) platensis já convertido em biomassa ( $\mathrm{g} / \mathrm{L}$ ). Para a conversão foi utilizada a Equação 1

Biomassa $(\mathrm{g} / \mathrm{L})=2,252 \times($ abs $)-0,508$

Gráfico 1. Resultado, do primeiro experimento, em biomassa ( $\mathrm{g} / \mathrm{L}$ ) de Arthrospyra (Spirulina) platensis em quatro tipos de garrafa de polietileno (azul, verde, vermelha e transparente) no decorrer 22 dias.

Graph 1. Result of the first experiment, in biomass $(g / L)$ of Arthrospyra (Spirulina) platensis in four types of polyethylene bottle (blue, green, red and transparent) over the course of 22 days.

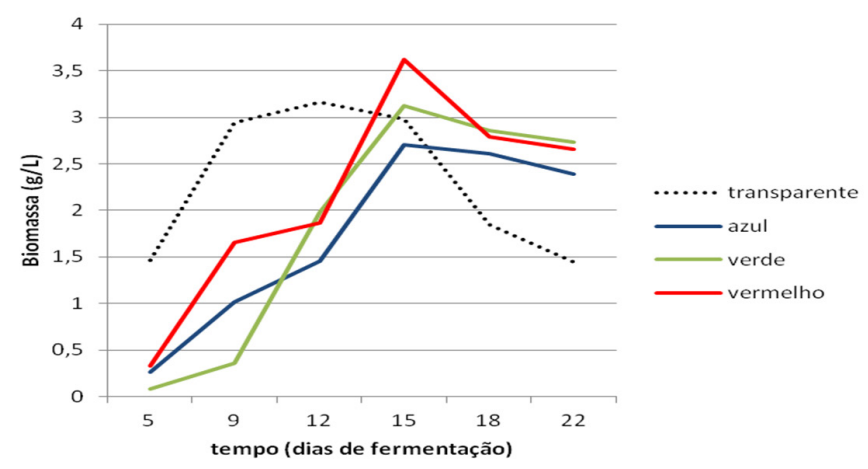

Gráfico 2. Resultado, do segundo experimento, em biomassa ( $g / L$ ) de Arthrospyra (Spirulina) platensis em quatro tipos de garrafa de polietileno (azul, verde, vermelha e transparente) no decorrer 22 dias.

Graph 2. Result, from the second experiment, in biomass $(g / L$ ) of Arthrospyra (Spirulina) platensis in four types of polyethylene bottle (blue, green, red and transparent) over the course of 22 days.

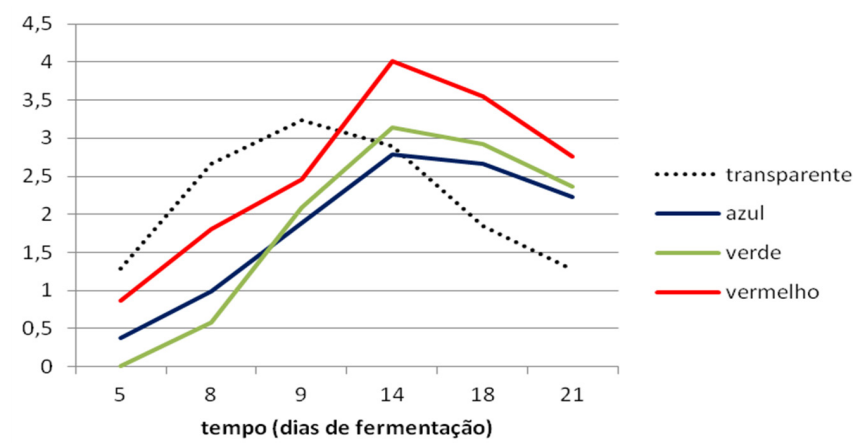

\section{DISCUSSÕES}

As análises feitas nos experimentos mostraram resultados distintos, entre as quatro cores utilizadas (transparente, azul, verde e vermelho), no intervalo de tempo de 22 dias.

O reator de cor azul foi o que teve menos crescimento e sua massa produzida de apenas $2,7011 \mathrm{~g} / \mathrm{L}$ no primeiro experimento e $2,792306 \mathrm{~g} / \mathrm{L}$ no segundo experimento. Como pode ser visto no trabalho de Souza et al. (2011), que aponta que o Guaco (Mikania laevigata) se desenvolve melhor e produz mais cloroplastos em ambiente com cobertura azul ou seja a planta teve que desenvolver mais cloroplastos para compensar a menor luminosidade proporcionada pela cobertura. Mas Oliveira (2014) relatou que a utilização de malha azul resultou maior produção de biomassa e teor de óleo essencial nas plantas, sendo, portanto, indicada sua utilização para o cultivo de melissa (Melissa officinalis L.).

O reator de cor verde teve uma produção de biomassa maior apenas que o reator de cor azul, produzindo apenas $3,125602 \mathrm{~g} / \mathrm{L}$ no primeiro experimento e $3,14024 \mathrm{~g} / \mathrm{L}$ no segundo experimento, também como na cor azul, a cor verde fez com que a planta aumentasse o número de cloroplastos para conseguir energia necessária para seu crescimento, de acordo com o trabalho de Queiroga et al. (2001), No caso de Arthrospyra (Spirulina) com o reator verde e azul houve uma diminuição da luminosidade que levou a uma menor produção da célula.

O reator transparente atingiu seu pico de crescimento mais rapidamente porém sua massa produzida foi de $3,165012 \mathrm{~g} / \mathrm{L}$ no primeiro experimento e $3,24158 \mathrm{~g} / \mathrm{L}$ no segundo experimento, sendo inferior do que o produzida pela cor vermelha, e seu morte foi mais rápida do que as demais cores. Segundo Souza et al. (2011) a grande intensidade da luz pode favorecer a degradação 
mais rápida. E de acordo com Oliveira et al. (2008), o principal fator que altera a atividade fotossintética é a luz, as telas coloridas alteram o espectro de luz transformando a luz em aumento de produtividade. No caso da Arthrospyra (Spirulina) o reator transparente não possui essa capacidade, frente ao reator vermelho.

Em trabalho realizado com tomate observou que a taxa de fotossíntese, condutância estomática e transpiração foram maiores nas plantas cultivadas sob luz com os comprimentos de onda na faixa do laranja, violeta e azul (PAIXÃO, 2014).

O reator de com vermelha foi o que mais teve crescimento no decorrer dos 22 dias, produzindo 3,617664g/L no primeiro experimento e 4,00726g/L no segundo experimento, pois tem a capacidade de alterar o espectro de luz e pode melhorar o mecanismo de fotossíntese de acordo com Henrique et al. (2011), esse fato também foi observado na produção de biomassa da Spirulina sp.

\section{CONCLUSÕES}

A coloração dos reatores afeta o crescimento e o desenvolvimento da Arthrospyra (Spirulina). Entre as quatro colorações de reatores estudadas, a de cor vermelha foi a mais eficiente na produção de Arthrospyra (Spirulina).

A cor vermelha atingiu um pico maior de crescimento e seu tempo de vida se mostrou maior, portanto a cor vermelha mostrou exercer influência sobre o crescimento da Arthrospyra (Spirulina) sp podendo assim, vir a ser utilizada na produção em alta escala.

\section{REFERÊNCIAS}

AMBROSI, M. A.; REINEHR, C. O.; BERTOLIN, T. E. et al. Propriedades de saúde de Spirulina spp. Journal of Basic and Applied Pharmaceutical Sciences, v. 29, n. 2, 2008. Disponível em: http://www.fcfar. unesp.br/revista_pdfs/vol29n2/trab1.pdf. Acesso em: 14 mar. 2014.

ARRUDA, R. O. M.; BRITO, A. W.; SILVA, R. R. et al. Fermentação de Spirulina platensis sob condições Naturais de Temperatura e Insolação. Revista Saúde-UnG. v. 3, n. 3, p.16-9. 2009. Disponível em: www.revistas.ung.br/index.php/saude/article/download/124/549. Acesso em: 25 mar. 2014.
BERTOLDI, F. C.; SANT'ANNA, E.; OLIVEIRA, J. L. B. Revisão: Biotecnologia de microalgas. Boletim do Centro de Pesquisa de Processamento de Alimentos, v. 26, n. 1, p. 9-20, 2008. Disponível em: http:// ojs.c3 sl.ufpr.br/ojs-2.2.4/index.php/alimentos/article/ view/11804/0. Acesso em: 22 jul. 2020.

BERTOLIN, T. B. P.; COSTA, J. A. V.; COLLA, L. M. et al. Cultivo da Cianobactéria Spirulina platensis a Partir de Efluente Sintético de Suíno. Ciênci agrotec, v. 29, n. 1, p. 118-25. 2005. Disponível em: http://www.scielo.br/pdf/ cargo/v29n1/a15.pdf. Acesso em: 14 mar. 2015.

BOROWITZKA, M. Commercial productiono of microalgae: ponds, tanks, tubes and fermenters. J. Biotechnol, v. 70. p.313-21. 1999.

BRASIL. Ministério da Saúde. Anvisa - Legislação Portaria $n^{\circ}$ 19, de 15 de março de 1995. DOU

DE 16/03/95 Anexo "I" Disponível em: http://www.anvisa.gov.br/legis/portarias/19_95.htm. Acesso em: 25 mar. 2014.

FRANCO, A. L. C.; LÔBO, I. P.; CRUZ, R. S. et al. Biodiesel de Microalgas: avanços e Desafios. Quim nova, v. 36, n. 3, p. 437-48. 2013. Disponível em: www.scielo.br/pdf/qn/v36n3/a15v36n3.pdf. Acesso em: 25 mar. 2014.

HENRIKSON, R. Microalga Spirulina, Superalimento del future. Barcelona: Ediciones S.A. Urano, 1994.

HENRIQUE, P. C.; ALVES, J. D. DEUNEL, S. et al. Aspectos fisiológicos do desenvolvimento de mudas de café cultivadas sob telas de diferentes colorações. Pesquisa Agropecuária Brasileira, v. 46, n. 5, p. 458465, 2011. Disponível em: http://www.scielo.br/pdf/pab/ v46n5/02.pdf. Acesso em: 22 jul. 2020

MEZZOMO, N.; SAGGIORATO, A. G.; SIEBERT, R. et al. Cultivation of microalgae Spirulina platensis (Arthrospira platensis) from biological treatment of swine wastewater. Food Science and Technology, v. 30, n. 1, p. 173-178, 2010. Disponível em: http://www.scielo.br/pdf/ cta/v30n1a26.pdf. Acesso em: 22 jul. 2020.

MOREIRA, R. L.; COSTA, J. M.; QUEIROZ, R. V. et al. 
Utilização de Spirulina platensis como suplemento alimentar durante a reversão sexual de tilápia do Nilo. Revista Caatinga, v. 23, n. 2, p. 134-141, 2010. Disponível em: http://www.periodicos.ufersa.edu.br/Índex.php/sistema. Acesso em: 22 jul. 2020.

MUliteRnO, A.; MOSELE, P. C.; COSTA, J. A. V. et al. Cultivo mixotrófico da microalga Spirulina platensis em batelada alimentada. Ciência e Agrotecnologia, v. 29, n. 6, p. 1132-1138, 2005. Disponível em: http://www. scielo.br/scielo.php. Acesso em: 22 jul. 2020.

OLIVEIRA, J. R. et al. Cultivos agrícolas utilizando telas coloridas e termorefletoras. Jornada Científica, v. 1, p. 5, 2008. Disponível em: https://www.bambui. ifmg.edu.br/jornada_cientifica/str/artigos_aprovados/ Ci\%c3\%aancias\%20Agrarias/34-PT-6.pdf. Acesso em: 22 jul. 2020.

OLIVEIRA, Graziele Cristina de. Photosynthetic, anatomical and productive behaviors of Melissa officinalis L. grounded under colored nets. 2014. $44 \mathrm{f}$. Dissertação (Mestrado em Ciências Agrárias) - Universidade do Oeste Paulista, Presidente Prudente, 2014. Disponível em: http://bdtd.unoeste.br:8080/jspui/handle/ tede/594. Acesso em: 22 jul. 2020.

PAIXÃO, Anna Carla de Castro. Efeito da luz transmitida através de coberturas coloridas transparentes sobre a mancha-alvo do tomateiro. 2014. 64 f. Dissertação (Agricultura no Trópico Úmido (ATU)) - Instituto Nacional de Pesquisas da Amazônia, Manaus, 2014. Disponível em: https://repositorio.inpa.gov.br/handle/1/5229. Acesso em: 22 jul. 2020.

QUEIROGA, R. C. F.; NETO, F. B.; NEGREIRO, M. Z. et al. Produção de alface em função de cultivares e tipos de tela de sombreamento nas condições de Mossoró. Horticultura Brasileira, v. 19, n. 3, p. 324-328, 2001. Disponível em: http://www.scielo.br/pdf/hb/v19n3/ v19n3a06. Acesso em: 22 jul. 2020.

RAVEN, P. H.; EVERT, R. F.; EICHHOM, S. E. Biologia Vegetal. 6. ed. Rio de Janeiro: Guanabara Koogan, 2001. technology and applied phycology. 21. Ed. USA: Blackwell Publishing, 2004.

SANTOS, G. M.; MACEDO, R. V. T.; ALEGRE, R. M. Influência do teor de nitrogênio no cultivo de Spirulina maxima em duas temperaturas-Parte I: Alteração da composição da biomassa. Food Science and Technology, v. 23, p. 17-21, 2003. Disponível em: http://www.scielo. br/scielo.php. Acesso em: 22 jul. 2020.

SASSANO, C. E. N.; CARVALHO, J. C. M.; CONVERTIL, A. et al. Estudo bioenergético do cultivo de Spirulina platensis utilizando uréia como fonte de nitrogênio. Resultados preliminares. Farmácia e Química, v. 35, n. 2, p. 37-43, 2002.

SATO, U. A. L. S. Proteínas de origem microbiana. In: Aquarone E, Borzani W, Schmidell W, Lima UA. Biotecnologia Industrial, v. 4, n. 3. p.457-60. 2009.

SILVA, S. M. C. S.; MURA, J. D. A. P.; AYOUB, M. E. Terapia Nutricional na Lipodistrofia Ginóide. In: Alimentação, Nutrição e Dietoterapia. São Paulo: Roca, 2007. p. 650-1.

SOUZA, G. S.; CASTRO, E. M.; SOARES, A. M. et al. Teores de pigmentos fotossintéticos, taxa de fotossíntese e estrutura de cloroplastos de plantas jovens de Mikania laevigata Schultz Bip. ex Baker cultivadas sob malhas coloridas. Semina: Ciências Agrárias, v. 32, n. supl 1, p. 1843-1854, 2011. Disponível em: http://www.ufrgs.br/ seerbio/ojs/index.php/rbb/article/view/1453. Acesso em: 22 jul. 2020.

VONSHAK, A. Spirulina Platensis, Physiology, Cell-Biology and Biotechnology. London: Taylore Francis, 1997. p.43-66.

RICHMOND, A. Handbook of microalgal culture: bio- 